\title{
Efecto de un programa de entrenamiento de fuerza sobre IGF-1 en adultos mayores con obesidad e hipertensión controlada \\ Effect of a strength training program on IGF-1 in older adults with obesity and controlled hypertension
}

*Rogelio Galaviz Berelleza, *Marina Trejo Trejo, *Juan Carlos Borbón Román, *Edgar Ismael Alarcón Meza, *Heriberto Antonio Pineda Espejel, *Emilio Manuel Arrayales Millan, ${ }^{* *}$ Guadalupe Simanga Robles Hernández, ${ }^{*}$ Lourdes Cutti Riveros

*Universidad Autónoma de Baja California (México), **Universidad Autónoma de Chihuahua (México)

\begin{abstract}
Resumen. Introducción: A medida que se presenta el envejecimiento, los músculos disminuyen su tamaño y fuerza. La pérdida de masa muscular puede causar una disminución general de la función metabólica asociada a una baja producción hormonal anabólica y riesgo de lesión. La disminución de los niveles séricos de GH e IGF-1 debido al incremento de la edad pueden promover la fragilidad al contribuir a la pérdida de masa muscular y fuerza objetivo: Determinar efecto de un programa de entrenamiento de fuerza sobre IGF-1 en adultos mayores con obesidad e hipertensión controlada. Método: 8 adultos mayores con obesidad e hipertensión controlada, realizaron 6 semanas de entrenamiento de la fuerza con intensidades del $70 \%$ del $1 \mathrm{RM}$, con una frecuencia de 4 veces por semana, previo al protocolo de entrenamiento se determinó triglicéridos, colesterol, glucosa e IGF-1 en sangre y medidas antropométricas (talla, peso, \% grasa y masa muscular). Se aplicó un test de una repetición máxima (1RM) para evaluar la fuerza máxima en el tren superior e inferior antes y después del programa de entrenamiento. Resultados: Se observó un aumento significativo $(\mathrm{p}<0.05)$ en los niveles séricos de IGF-1, después de la realización del programa de entrenamiento. Conclusión: El entrenamiento de la fuerza con intensidades del 70\% de la 1RM y frecuencia 4 realizado durante 6 semanas en adultos mayores con obesidad e hipertensión controlada, produjo incrementos en la concentración sérica de IGF-1 y un incremento de la fuerza muscular.
\end{abstract}

Palabras Clave: IGF-1, Adulto Mayor, Sarcopenia, Entrenamiento.

Abstract. Introduction: As people age, muscles decrease in size and strength. Loss of muscle mass can cause a general decrease of metabolic function associated with low anabolic hormone production and risk of injury. The decrease in serum levels of GH and IGF-1 due to aging can provoke fragility by contributing to the loss of muscle mass and strength. Purpose: To determine the effect of a strength training program on IGF-1 in older adults with obesity and controlled hypertension. Method: a sample of 8 older adults with obesity and controlled hypertension performed 6 weeks of strength training with intensities of 70\% 1RM and 10RM, with a frequency of 4 times per week. Prior to the protocol of Training, triglycerides, cholesterol, blood glucose IGF-1, and anthropometric measures (size, weight, $\%$ fat and muscle mass) were determined. The test of one maximum repetition ( $1 \mathrm{rm})$ was performed to evaluate maximum force in upper and lower limbs before and after the training program. Results: A significant increase $(p<0.05)$ was observed in serum IGF-1 levels after the completion of training program. Conclusion: Strength training with intensities of $70 \%$ of 1RM and frequency of 4 days/week, carried out for 6 weeks, produced increases in serum IGF-1 concentration and increased muscle strength in older adults with obesity and controlled hypertension.

Keywords: IGF-1, Seniors, Sarcopenia, Training.

\section{Introducción}

El Factor de crecimiento 1 similar a la insulina (IGF-1) es un importante biomarcador metabólico asociado con una variedad de resultados relacionados con la salud y el ejercicio (Nindl,2010), tiene un efecto anabólico importante en la síntesis de proteínas y mantenimiento o mejora de la masa muscular, es particularmente importante en poblaciones de mediana y mayor edad que se encuentran en riesgo de pérdida de masa muscular (Kraemer \& Castracane, 2015). El IGF-1 está implicado en la vía final común de algunas hormonas como la hormona de crecimiento $(\mathrm{GH})$, los esteroides sexuales, glucocorticoides y las hormonas tiroideas, su disminución se ha relacionado con múltiples enfermedades como la baja talla, el síndrome de insensibilidad a la hormona del crecimiento y la diabetes mellitus entre otros (Cadena, Cornejo, Pinto, Fabián \& Rueda,1998). Las concentraciones de IGF-1 aumentan gradualmente en la infancia y la pubertad, mientras que disminuyen durante el envejecimiento (Martinelli, Custodio, \& Aguiar-Oliveira, 2008).

Fecha recepción: 17-10-19. Fecha de aceptación: 17-06-20

Marina Trejo Trejo

marina.trejo@uabc.edu.mx
Existe evidencia de la correlación significativa entre el cambio porcentual en IGF-I libre y el porcentaje de cambio en masa corporal y masa libre de grasa. De hecho, el IGF-I se asocia con alteraciones y/o cambios en la composición corporal, estos cambios se deben a la influencia somatotrófica que ejerce IGF-I en los tejidos ya que ejerce acciones anabólicas, mitogénica y miogénica tanto en una endócrina como en un modo autócrino y / o parácrino ((Nindl et al., 2007). Sung, Moon, Hyo y Jin (2017), encontraron una disminución en la concentración de IGF-1 al realizar ejercicio de resistencia y restricción calórica que se correlacionaba con la disminución de masa muscular. Se ha descrito correlación entre niveles de IGF-I y los cambios en tanto en masa corporal y masa libre de grasa observándose que con un decremento en la concentración de IGF-1 libre indudablemente refleja una capacidad de síntesis y mitótica disminuida para mantener el recambio y equilibrio de las proteínas, lo cual se refleja por la pérdida de masa libre de grasa (Nindl et al, 2007).

El adulto mayor es común que presente patologías como hipertensión, diabetes, obesidad entre otros, esto debido propiamente al proceso de envejecimiento (Da Costa, Vitorino, Silva, Vogel, Duarte \& Rocha-Santos, 2016) así como disminución de sus funciones fisiológicas (Trejo et al., 2018), 
y así mismo se presentan alteraciones hormonales como la disminución de la actividad de la hormona de crecimiento (GH) y el eje GH / IGF-1, lo que resulta en una concentración reducida de IGF-1 y una reducción en la función de la vía de señalización de IGF-1 (Brown-Borg, Borg, Melsika, \& Bartke, 1996; Milman et al., 2014; Van der Spoel et al. 2015), además existe un declive progresivo de la masa y la función del músculo esquelético (sarcopenia), y la pérdida de masa libre de grasa (Roubenoff et al., 2003). La sarcopenia, se asocia con varios resultados adversos, incluyendo pérdida de autonomía y mortalidad. La evidencia sugiere la existencia de una relación entre los niveles de la hormona del crecimiento $(\mathrm{GH})$ y del factor de crecimiento similar a la insulina-1 (IGF-1) con los cambios en la composición corporal y en la función física, por lo tanto, la disminución en los niveles séricos de GH y IGF-1 dependiente de la edad pueden promover la fragilidad al contribuir a la pérdida de masa muscular y fuerza (Nicklas et al., 1995; Roberts et al., 2010; Weltman et al., 1997).

El entrenamiento de la fuerza representa actualmente la estrategia terapéutica primaria más recomendada para prevenir y revertir el declive de la masa muscular, fuerza y funcionalidad asociado a la edad (Castro, Gálvez, Guzmán, \& Garcia, 2019; Izquierdo et al., 2001). Desde mediados de los años 80 se han desarrollado numerosos estudios sobre los efectos del entrenamiento de fuerza sobre distintas medidas de la fuerza y morfología muscular en adultos mayores. Algunos de esos estudios de intervención han comparado entre sí distintos protocolos de entrenamiento para comprobar las ganancias de fuerza máxima, fuerza explosiva, potencia y capacidad funcional (Ramírez-Campillo et al, 2014; Seynnes, etal., 2004).

Unos de los factores críticos para el anabolismo del musculo esquelético cuando se realiza ejercicio físico son las respuestas hormonales que incluyen testosterona, hormona del crecimiento (GH) e IGF-1, si bien el IGF-1 es producido sistemáticamente por el hígado de manera endocrina, este también se produce localmente en el músculo esquelético para actuar de manera autocrina/paracrina. El IGF-1, producido en el músculo esquelético durante el ejercicio también es liberado hacia la circulación y podría contribuir a un aumento en los niveles circulantes de IGF-1 (Berg \& Bang, 2014; Berg, Salitin, \& Hall, 1997)

Por lo anteriormente señalado los regímenes de ejercicio pueden ser prescritos para mejorar respuestas hormonales y así mejorar las adaptaciones del músculo esquelético como fuerza y ganancia de masa muscular en el adulto mayor. El propósito del estudio fue determinar efecto de un programa de entrenamiento de fuerza sobre la concentración sérica de IGF-1 en adultos mayores con obesidad e hipertensión controlada.

\section{Materialy Métodos}

\section{Diseño}

Este estudio fue Cuantitativo, prospectivo, transversal y cuasiexperimental.

\section{Participantes}

Participaron 8 hombres que contaban con los criterios de inclusión para participar en el estudio que fueron mayores de 60 años (61.6 \pm 1 años), sedentarios con obesidad e hipertensión controlada. los participantes firmaron un consentimiento informado y se observaron estrictamente los aspectos éticos de protección de los pacientes, bajo lo estipulado en el Código de Helsinki y la Ley General de Salud de México y aprobado por el comité de ética de la facultad de deportes. Los participantes fueron excluidos del estudio si: Sufrieron algún tipo de lesión neuromuscular o faltaron a dos o más sesiones consecutivas. Los participantes podían retirarse del estudia cuando ellos lo quisieran.

\section{Toma de muestras sanguíneas y parámetros clínicos}

Los sujetos fueron citados en el laboratorio de Fisiología del ejercicio de la facultad de deportes de la Universidad Autónoma de Baja California para la realización de historia clínica y presión arterial. Muestras de sangre fueron colectadas de vena antecubital en posición sedente para la determinación glucosa, colesterol, triglicéridos a través de método enzimático (Spin React, Modelo SPIN120, SP) e IGF-1 (Grupo Diagnóstico PROA S.A. de C.V., laboratorio internacional de referencia) antes del inicio del programa de entrenamiento de fuerza y al finalizar la implementación del programa.

\section{Determinación de composición corporal}

Las mediciones de composición corporal se realizaron con mínimo 2 horas de ayuno y sin haber realizado actividad física. La talla se determinó por medio de un estadímetro (SECA, modelo 213, Seca Corp, Hanover, MD, USA), el peso, $\%$ de grasa y $\%$ de masa muscular se realizó por medio de técnica de impedancia bioeléctrica (inbody modelo 720 marca Biospace, Hennock Road East, Marsh Barton, UK), realizándose las mediciones con un ayuno mínimo de 2 .

\section{Evaluación de fuerza máxima (1RM)}

La fuerza máxima fue valorada por medio del test de 1 repetición máxima (1RM) que consiste en el vencimiento de la mayor carga que pudiera ser levantada correctamente una vez en press de pierna y press de banca, comenzando con un calentamiento consistió de una serie de 8-10 repeticiones al 40-60\% del máximo estimado a través del peso corporal, posteriormente los sujetos descansaron por un minuto, después de esto, se realizaron de 3-5 repeticiones con el 60-80\% del máximo estimado. Para determinar la 1RM se realizaron de tres a cuatro intentos con una pausa de 3-5 min entre los levantamientos. Para que cada prueba de 1RM se considerara exitosa se requirió que en el ejercicio press de pierna los participantes colocaran los pies en la plataforma con una separación igual a la de los hombros y en un ángulo de 90 grados con las rodillas y a continuación el participante empujó contra la plataforma hasta que las piernas estuvieron en extensión (sin bloquear las rodillas) volviendo a la posición inicial. Para que la prueba de press de banca fuera válida los participantes debían tener los pies en todo momento haciendo contacto con el suelo, los glúteos, la parte alta de la espalda y la cabeza haciendo contacto con la banca. El agarre de la barra lo hicieron igual a la separación de los hombros. Desde esa posición los participantes retiraban la barra del rack con ayuda de un monitor, y entonces se bajaba la barra hasta que hicieran contacto con los pectorales, y finalmente debían regresar a la posición inicial con los brazos en com- 
pleta extensión y los codos perfectamente bloqueados. Durante la evaluación de la 1RM no se produjeron lesiones.

\section{Programa de entrenamiento}

El entrenamiento se llevó a cabo por 6 semanas y se implementó el programa de ejercicios 4 días por semana de los cuales dos días se realizaron trabajo en el tren superior (lunes y jueves) y otros dos días se realizó trabajo del tren inferior (martes y viernes). Para el trabajo de tren superior se llevaron a cabo 4 ejercicios los cuales se realizaron a través de una tracción horizontal (remo en maquina), una tracción vertical (jalón al pecho) un empuje horizontal (prensa de banca), un empuje vertical (prensa militar) y en los 4 ejercicios del tren inferior que involucraron a los extensores de rodilla (prensa de pierna, extensiones de pierna), extensores de cadera (curl femoral), dorsiflexores (elevaciones de talones sentado y de pie con barra) realizados en máquinas isotónicas (Stand, 2009; Izquierdo 2001; Henwood et al., 2008; Pereira et al., 2012).

En las primeras 2 semanas del programa de entrenamiento se llevó a cabo una adaptación anatómica donde se incluyeron cargas del 40\% del 1-RM para el tren superior y del $60 \%$ de su 1-RM para el tren inferior con un volumen de 3 series de 15 repeticiones para todos los ejercicios, con una densidad de 60 segundos entre serie y 120 segundos entre ejercicios (Chicharro y Mojares, 2008).

Posteriormente los sujetos entrenaron durante 4 semanas a un $70 \%$ de su 1-RM en los ejercicios de press de pierna y press de banca y a su 1-RM en el resto de ejercicios. $\mathrm{Su}$ volumen de entrenamiento fue de 4 series de 10 repeticiones, con una densidad de entre 60 y 90 segundos entre series y 120 a 180 segundos entre ejercicio (Schoenfeld, B. J., Ogborn, D., \& Krieger, J. W. (2016).

\section{Análisis estadístico}

Las variables fueron analizadas con medidas de tendencia central y de dispersión. Para evaluar el efecto del entrenamiento de fuerza se utilizó estadística inferencial aplicando una prueba t de Student. Los datos fueron analizados en el software IBM SPSS Statistics versión 22. Se consideró como valor significativo $p<0.05$.

\section{Resultados}

Las características antropométricas y fisiológicas de los sujetos se describen en la tabla 1, observándose parámetros recomendables a excepción del IMC (índice de masa corporal).

Los resultados de la prueba de press de banca indican que hubo cambios significativos $(p<0.05)$ ( $1 \mathrm{RM}$ de $52.5 \pm 26.4$ $\mathrm{kg}$ inicial contra un $1 \mathrm{RM}$ de $58.3 \pm 22 \mathrm{~kg}$ final), (Figura 1a). El resultado en el press de pierna inicial fue de $1 \mathrm{RM}$ de $154 \pm 79.9$ $\mathrm{kg}$ y posterior a la implementación del programa de entrenamiento de fuerza fue de $175.3 \pm 79.3 \mathrm{~kg}$., lo anterior refleja un aumento significativo en el 1RM de los participantes en press de pierna $(p<0.5)$ (Figura 1b).

Los resultados de los cambios en el peso corporal, $\% \mathrm{de}$ masa muscular y grasa se muestran en la tabla 2. En los 3 marcadores de composición corporal no se presentaron cambios estadísticamente significativos después de la
Características generales, de composición corporal e indicadores bioquímicos de los participantes

\begin{tabular}{cc}
\multicolumn{1}{l}{ participantes } & \\
\hline Características & M DS \\
\hline Edad (años) & $61.6 \pm 1$ \\
Peso $(\mathrm{kg})$ & $97.0 \pm 14.9$ \\
Talla $(\mathrm{cm})$ & $169 \pm 0.4$ \\
IMC $(\mathrm{kg} / \mathrm{m})$ & $34.1 \pm 3.7$ \\
TAS $(\mathrm{mmHg})$ & $122.0 \pm 7.0$ \\
TAD $(\mathrm{mmHg})$ & $83.5 \pm 9.6$ \\
$\% \mathrm{MM}$ & $36.5 \pm 1.5$ \\
Glucosa $(\mathrm{mg} / \mathrm{dl})$ & $86.4 \pm 18.6$ \\
Colesterol (mg/dl) & $104.3 \pm 17.3$ \\
Triglicéridos (mg/dl) & $124.9 \pm 29.3$ \\
\hline IMC: Índice de Masa Corporal; TAS: Tensión Arterial Síśólica; TAD: Tensión Arterial \\
Diástólica; MM: Masa Muscular; M: media; DS: desviación estándar.
\end{tabular}

))

b)
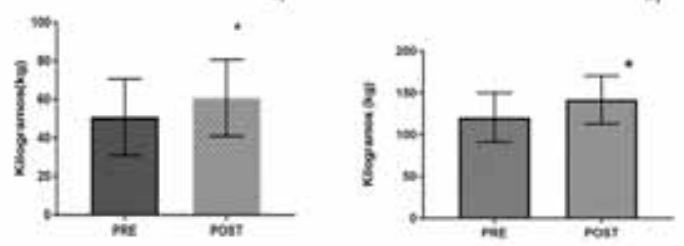

Figura 1. a) Efecto del entrenamiento de fuerza sobre 1RM en press de banco b) efecto del entrenamiento de fuerza sobre $1 \mathrm{RN}$ en press de pierna. ${ }^{*}=p<0.05$. Tabla 2.

Cambios en la composición corporal de los sujetos

\begin{tabular}{lccc}
\multicolumn{4}{l}{ Cambios en la composición corporal de los sujetos } \\
\hline Caracteristicas & $\pm \mathrm{DS}$ & $\pm \mathrm{DS}$ & $p$ \\
\hline Peso $(\mathrm{kg})$ & $97.0 \pm 14.9$ & $96.0 \pm 14.8$ & $>0.05$ \\
\% Masa Muscular & $36.5 \pm 1.5$ & $37.1 \pm 1.1$ & $>0.05$ \\
\% Masa Grasa & $35.5 \pm 2.8$ & $34.5 \pm 2.5$ & $>0.05$ \\
\hline
\end{tabular}
$\%$ Masa Grasa

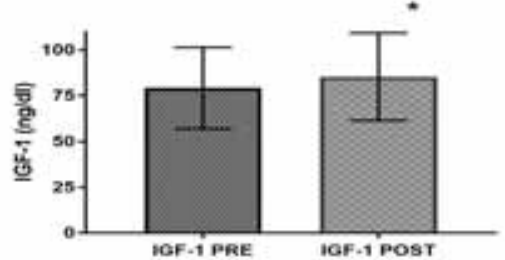

Figura 2. Cambios en el IGF-1. ${ }^{*} p<0.05$

implementación del programa de entrenamiento. $(p>0.05)$.

Las modificaciones en la concentración sérica de IGF-1 de los sujetos se muestran en la figura 2. La concentración inicial de IGF-1 (79.2 $\pm 22.2 \mathrm{ng} / \mathrm{dL})$ y las finales $(83.7 \pm 23.8 \mathrm{ng} /$ dL) observándose un aumento estadísticamente significativo después de la en los niveles de IGF-1 en los sujetos fue significativo $(p<0.05)$.

\section{Discusión}

Los resultados del presente estudio no muestran una diferencia significativa en la composición corporal de los sujetos después de la realización del entrenamiento de fuerza de 6 semanas ( $p>0.05$ ), esto es, no se presentó modificaciones en el peso corporal y porcentaje de grasa corporal así como en porcentaje de masa muscular, al respecto, otros estudios nos describen que las diferencias significativas en la composición corporal se observan a partir de 8 semanas de entrenamiento Chen, Chung, Chen, Ho, \& Wu, 2017; Sarsan, Ardiç, Özgen, Topuz, \& Sermez, 2006).

En la fuerza máxima evaluada por medio de $1 \mathrm{RM}$ en press de banco y de pierna se observó un incremento significativo después de la aplicación del programa de entrenamiento a pesar de no presentarse incremento de masa muscular y al respecto es bien sabido que la magnitud de la fuerza voluntaria máxima, al igual que el rendimiento en el levantamiento de pesas se encuentran estrechamente relacionados con el área transversal del músculo, sin embargo la expresión de la fuerza tiene un componente neuronal significativo.

Los niveles sanguíneos de IGF-1 de los participantes 
mostraron un aumento significativo $(p<0.05)$ después de la realización de un entrenamiento de fuerza de 6 semanas de duración, mientras que Roberts et al. (2010) no observó diferencias entre los niveles de IGF-1 entre adultos mayores luego de entrenamiento de fuerza de 12 días a una intensidad del $80 \%$ de la 1RM lo cual indica que periodos tan cortos de entrenamiento no tienen influencia en los niveles de IGF-1. El comportamiento de la hormona IGF-1 en nuestro estudio fue significativamente más alto después de la realización del programa de entrenamiento. sin embargo, aunque no se observó un aumento en la masa muscular esto probablemente por el corto tiempo del programa, y se podría explicar por lo señalado por lo podría explicar Drummond et al. (2009), que muestra evidencias que sugieren: 1) la expresión del péptido de IGF-1 es mínimamente afectada con el envejecimiento y 2) una desconexión molecular entre IGF-1 receptor en el músculo esquelético (es decir, el IGF-1receptor de IRS-1 mediada por el receptor) teniendo más relevancia en relación con el desarrollo de la sarcopenia.

\section{Conclusión}

El entrenamiento de la fuerza con intensidades del $70 \%$ de la $1 \mathrm{RM}$ y frecuencia 4 realizado durante 6 semanas en adultos mayores con obesidad e hipertensión controlada, produjo incrementos en la concentración sérica de IGF-1 y un incremento de la fuerza muscular.

\section{Referencias}

Berg, U., Salitin, B. y Hall, K, (1997). Net fluxes of IGF-1, IGFBP-1, insulin, GH and energy substrates over contracting muscle during prolonged exercise in healthy men. Endocrinoogy and Metabolism. 4, 313-320.

Berg, U. y Bang, P. (2014). Exercise and circulating insulin-like growth factor I. Hormone Researchs in Pediatrics. 62 (Suppl 1) 50-58.

Brown-Borg, H. M., Borg, K.E., Melsika, C.J., \& Bartke. A. (1996). Dwarf mice and the ageing process. Nature, 384: 33.

Cadena Ortiz, L. M., Cornejo Martínez, S. J., Pinto, Q., Fabián, M., \& Rueda Ochoa, O. L. (1998). Factor de crecimiento similar a la insulina: nuevos avances y perspectivas terapéuticas. MedUNAB, 1(3), 204208.

Castro, L.E., Gálvez, A.Y., Guzmán, G.A., Garcia, A. (2019) Fuerza explosiva en adultas mayores, efectos del entrenamiento en fuerza máxima. RETOS, 36, 64-68.

Chen, H.-T., Chung, Y.-C., Chen, Y.-J., Ho, S.-Y., \& Wu, H.-J. (2017). Effects of Different Types of Exercise on Body Composition, Muscle Strength, and IGF-1 in the Elderly with Sarcopenic Obesity. Journal of the American Geriatrics Society, 65(4), 827-832. doi:10.1111/ jgs. 14722.

Chicharro, J. L. \& Mojares, L. M. L. (2008). Fisiología clínica del ejercicio. Ed. Médica Panamericana.

Da Costa, J. P., Vitorino, R., Silva, G. M., Vogel, C., Duarte, A. C., \& Rocha-Santos, T. (2016). A synopsis on aging-Theories, mechanisms and future prospects. Ageing Research Reviews, 29, 90-112.

Drummond, M. J., Miyazaki, M., Dreyer, H. C., Pennings, B., Dhanani, S., Volpi, E., ... \& Rasmussen, B. B. (2009). Expression of growthrelated genes in young and older human skeletal muscle following an acute stimulation of protein synthesis. Journal of Applied Physiology, 106(4), 1403-1411.

Henwood, T.R., Riek, S., \& Taaffe, D.R. (2008). Strength versus muscle power-specific resistance training in community-dwelling older adults. Journal of Gerontology. A Biol. Sci. Med. Sci. 63, 83-91.

Izquierdo, M., Hakkinen, K., Ibanez, J., Garrues, M., Anton, A., Zuniga, A., Larrion, J.L. y Gorostiaga, E.M. (2001). Effects of strength training on muscle power and serum hormones in middle-aged and older men. Journal of Applied Physiology. 90, 1497-1507.

Kraemer R.R. y Castracane, V.D. (2015). Endocrine alterations from concentric vs. eccentric muscle actions: a brief review. Metabolism, 64, 190-201.

Martinelli, C. Jr., Custodio, R.J. \& Aguiar-Oliveira, M.E. (2008). Physiology of the GH-IGF axis. Arquivos Brasileiros de Endocrinologia \& Metabologia, 52, 717-725.

Milman, S., Atzmon, G., Huffman, D.M., Wan, J., Crandall, J.P., Cohen, P. \& Barzilai, N. (2014). Low insulin-like growth factor-1 level predicts survival in humans with exceptional longevity, Aging Cell, 13: 769-71. International Journal of Sports Medicine, 16: 445-450.

Nindl, B.C., Alemany, J.A., Kellogg, M.D., Rood, J., Allison, S.A., Young, A.J. \& Montain S.J. (2007). Utility of circulating IGF-I as a biomarker for assessing body composition changes in men during periods of high physical activity superimposed upon energy and sleep restriction. Journal of Applied Physiology,103(1): 340-6.

Nindl, B.C. (2010). Insulin-like growth factor-I, physical activity, and control of cellular anabolism, Medicine \& Science in Sports \& Exercise, 42, 35-38.

Pereira, A., Izquierdo, M., Silva, A.J., Costa, A.M., Bastos, E., GonzalezBadillo, J.J., Marques, M.C. (2012a). Effects of high-speed power training on functional capacity and muscle performance in older women. Experimental Gerontology. 47, 250-255.

Pereira, A., Izquierdo, M., Silva, A.J., Costa, A.M., Gonzalez-Badillo, J.J., Marques, M.C., (2012b). Muscle performance and functional capacity retention in older women after high-speed power training cessation. Experimental Gerontology. 47, 620-624.

Roubenoff, R., Parise, H., Payette, H. A., Abad, L. W., D’Agostino, R., Jacques, P. F., ... \& Harris, T. B. (2003). Cytokines, insulin-like growth factor 1 , sarcopenia, and mortality in very old communitydwelling men and women: the Framingham Heart Study. The American Journal of Medicine, 115(6), 429-435.

Roberts, M. D., Dalbo, V. J., Sunderland, K. L., Poole, C. N., Hassell, S. E., Bemben, D., ... \& Kerksick, C. M. (2010). IGF-1 splice variant and IGF-1 peptide expression patterns in young and old human skeletal muscle prior to and following sequential exercise bouts. European Journal of Applied Physiology, 110(5), 961-969.

Ramírez-Campillo, R., Castillo, A., de la Fuente, C.I., Campos-Jara, C., Andrade, D.C., Álvarez, C., ... \& Izquierdo, M. (2014). High-speed resistance training is more effective than low-speed resistance training to increase functional capacity and muscle performance in older women. Experimental Gerontology, 58, 51-57.

Sarsan, A., Ardiç, F., Özgen, M., Topuz, O., \& Sermez, Y. (2006). The effects of aerobic and resistance exercises in obese women. Clinical Rehabilitation, 20(9), 773-782. doi:10.1177/0269215506070795

Seynnes, O., Fiatarone Singh, M. A., Hue, O., Pras, P., Legros, P., \& Bernard, P. L. (2004). Physiological and functional responses to low-moderate versus high-intensity progressive resistance training in frail elders. The Journals of Gerontology Series A: Biological Sciences and Medical Sciences, 59(5), M503-M509.

Stand, P. (2009). Progression models in resistance training for healthy adults. Medicine and Science in Sports and Exercise, 41(3), 687708 .

Schoenfeld, B. J., Ogborn, D., \& Krieger, J. W. (2016). Effects of resistance training frequency on measures of muscle hypertrophy: a systematic review and meta-analysis. Sports Medicine, 46(11), 1689-1697.

Sung, J., Moon, J., Hyo, M. \& Jin, S. (2017). Effect of low-intensity resistance training with heat stress on the HSP72, anabolic hormones, muscle size, and strength in elderly women. Aging Clinical and Experimental Research,29(5):977-984.

Trejo, M., Cisneros, F. J. D., Araujo, C. K., Cervantes, M. H. M., Garcidueñas, M. M. N., Guridi, R. C. A., \& Espejel, H. A. P. (2018). Efecto del ejercicio máximo y submáximo sobre excreción de albúmina en adultos mayores. Retos: nuevas tendencias en educación física, deporte y recreación, (33), 238-240.

Van der Spoel, E., Rozing, M.P., Houwing-Duistermaat, J.J., Slagboom, P.E., Beekman, M., de Craen, A.J.M., ... \& van Heemst, D. (2015). Association analysis of insulin-like growth factor-1 axis parameters with survival and functional status in nonagenarians of the Leiden Longevity Study, Aging, 7, 956-63.

Weltman, A., Weltman, J.Y., Womack, C.J., Davis, S.E., Blumer, J.L., Gaesser, G.A. \& Hartman, M.L. (1997). Exercise training decreases the growth hormone $(\mathrm{GH})$ response to acute constant-load exercise. Medicine \& Science in Sports \& Exercise, 29, 669-676. 